S. Chen - J. C. Fetzer $\cdot$ M. E. Meyerhoff

\title{
Retention behavior of large polycyclic aromatic hydrocarbons on metalloprotoporphyrin-silica stationary phases
}

Received: 21 June 2000 / Revised: 22 August 2000 / Accepted: 23 August 2000

\begin{abstract}
The retention behavior of large polycyclic aromatic hydrocarbons (LPAHs) ( $\geq 7$ rings) on newly developed metalloprotoporphyrin (MProP)-silica stationary phases is examined and the results are compared to previously reported data for retention of the same solutes on commercially available phases. HPLC columns packed with FeProP-silica are shown to exhibit unique shape selectivity for LPAH retention, with the planar LPAHs always retained much longer than corresponding non-planar solutes. Solute planarity, length to breadth ratio (L/B value), and number of carbon atoms within the LPAHs are all demonstrated to contribute to the retention sequence observed. Further, the retention of LPAH solutes on FeProP-silica phases is shown to be more predictable than on other reversed-phase columns, with the elution sequence constant regardless of the mobile phase composition. Due to the extremely high planar selectivity of $\mathrm{Fe}-$ ProP-silicas with respect to LPAH retention, it is envisioned that columns packed with these phases could be used in conjunction with existing commercial columns to devise methods for more efficient separation of complex mixtures of LPAHs in environmental and other samples.
\end{abstract}

\section{Introduction}

Large polycyclic aromatic hydrocarbons (LPAHs) are polycyclic aromatic hydrocarbons having seven or more conjugated aromatic rings. These compounds can be found in

S. Chen · M. E. Meyerhoff ( $\varangle)$

Department of Chemistry, The University of Michigan,

Ann Arbor, MI 48109-1055, USA

e-mail: mmeyerho@umich.edu

J. C. Fetzer

Chevron Research and Technology Company,

Richmond, CA 94802, USA

Current address:

S. Chen

Merck and Co., Inc., POB 2000, Rahway, NJ 07065-0900, USA diesel particulate extract, oil produced in catalytic hydrocracking, carbon black extract, and coal tar pitch [1-4]. The analysis of LPAHs is of great importance because of increasing concern over the mutagenicity and carcinogenicity of several of them. The level of the mutagenicity or carcinogenicity of a particular LPAH depends on its structure. Quantitation of the LPAHs with different structures and their present levels in various sources would be necessary prior to estimating their bioactivity. It is known that similar LPAH isomers can range from being very highly active to being totally inactive [5]. Many analytical techniques have been used for small PAH analysis, but many of the more common ones suffer from limitations when applied to LPAHs. The difficulty arises in precisely identifying the LPAH because the existence of numerous isomers increases when the number of rings increases. Driven by the unique nature of LPAHs, analytical techniques and approaches to the analysis remains an expanding area. Steric interactions between hydrogen atoms on adjacent rings force many of these compounds into threedimensional orientations, different from the planar structure someone might instinctively assume. Crystallography [6] and molecular modeling studies [3] have shown that certain LPAHs can be 30 degrees or more out of plane. Thus, these three-dimensional structures produce various unexpected ranges of chromatographic and spectral behaviors.

The complex nature of LPAH structures and the many possible isomers usually require separation techniques to be initially applied before other analytical methods. It is clear that as the number of rings grows, the number of possible isomers increases, and thus the analysis of LPAHs is more difficult than of smaller PAHs. Most LPAHs are not volatile and are, therefore, not easily amenable to analysis by gas chromatography (GC). The large number of isomers for LPAHs with same carbon number limits the use of mass spectrometry (MS), because of the identical molecular weights and similar fragmentation patterns that isomers could yield. High performance liquid chromatography (HPLC) has therefore become the preferred method for the separation and analysis of LPAHs. 
In HPLC, chemically bonded stationary phases are widely used as column packing materials. Such bonded phases have been prepared and applied to a great variety of separation problems with a high degree of success. Much research has focused on the separation of LPAHs on chemically bonded reversed-phases. Such phases are already used widely for the separation of small PAHs [7-14]. While researchers have demonstrated some success in using octadodecylsilyl(ODS)-silica phases in LPAH separations, usually sophisticated solvent programs and high temperatures are needed [15] because the difference in capacity factors of LPAHs on ODS phases is too large to use a single-solvent system or even a two-solvent system. This has led researchers to believe that the separation of LPAHs is much more complicated than the sep-

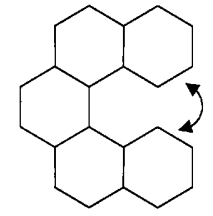

A

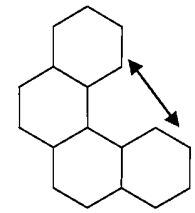

B

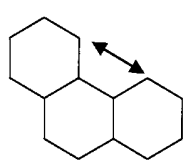

C
Fig. 1 Substructures of PAHs producing steric hindrance with respect to the final shape of the molecule: $(A)$ fjord, $(B)$ cove and $(C)$ bay aration of smaller PAHs as a result of the unique threedimensional conformations assumed by these molecules. $\pi$-Rich stationary phases such as diphenyl- or triphenylsilica phases have been studied [13,14]. The retention of LPAHs on these phases is somewhat more predictable than the retention behavior on polymeric ODS phases. This might be due to the fact that on diphenyl and triphenyl-silica, the immobilized ligand creates a more defined space for LPAH interaction than on the ODS phases.

Fetzer and Biggs have investigated the retention behavior of LPAHs on various bonded phases [15]. They have concluded that the nature of the stationary phase, together with the structural changes the stationary phase undergoes with different mobile phases, are very important to the final separation. Additionally, the characteristics of the given LPAH solute are also important to the final retention. Indeed, it was concluded that one of the most important factors is the shape of the LPAH. Three distinct retention behaviors can be linked to three classes of molecular shape. The first class of retention behavior includes LPAH solutes that remain planar in all solvent systems (Fig. 1 C). Examples of this class are coronene (B), dibenzo[cd,lm]perylene (A), benzo[pqr]naphtho[8,1,2-bcd]perylene (D), and naphtho[8,1,2-abc]coronene (G) (see Table 1 for LPAH structures examined in this work and corresponding identifying letters). These molecules are retained in a manner correlated with the number of $\pi$-elec-

Table 1 Capacity factors of LPAHs on FeProP-silica

\begin{tabular}{|c|c|c|c|c|c|c|c|}
\hline \multirow{2}{*}{\multicolumn{2}{|c|}{ Full name of LPAH }} & \multirow[t]{2}{*}{ Compound } & \multirow[t]{2}{*}{$\mathrm{L} / \mathrm{B}$} & \multirow[t]{2}{*}{ Ring \# } & \multicolumn{3}{|c|}{ Capacity factors } \\
\hline & & & & & $\mathrm{ACN}$ & Toluene & $\begin{array}{l}80 \% \text { ACN } \\
20 \% \text { toluene }\end{array}$ \\
\hline & Dibenzo[cd,lm]-perylene (planar) & A & 1.78 & 7 & 13.4 & 1.4 & 5.8 \\
\hline & Coronene (planar) & B & 1.17 & 7 & 11.0 & 1.2 & 5.3 \\
\hline & Dibenzo[j,lm]naphtho-[1,8-ab]perylene (twist) & $\mathrm{C}$ & 1.42 & 8 & 28.0 & 1.5 & 6.6 \\
\hline & Benzo[pqr]naphtho-[8,1,2-bcd]perylene (planar) & $\mathrm{D}$ & 1.36 & 8 & 44.0 & 3.2 & 15.1 \\
\hline & Pyranthrene (planar) & $\mathrm{E}$ & 1.78 & 8 & 49.0 & 3.5 & 17.5 \\
\hline & Benzo[lm]phenanthro-[4,5,6-abcd]perylene (twist) & $\mathrm{F}$ & 1.40 & 9 & 25.0 & 1.6 & 7.2 \\
\hline & Naphtho[8,1,2-abc]-coronene (planar) & G & 1.18 & 9 & 62.0 & 6.4 & 18.4 \\
\hline 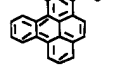 & Tetrabenzo[a,cd,j,lm]-perylene (non-planar) & $\mathrm{H}$ & 1.28 & 9 & 4.0 & 0.6 & 1.3 \\
\hline Eivio & Dibenzo[a,rst]naphtho-[8,1,2-cde]pentaphene (twist) & I & 1.57 & 9 & 24.0 & 1.6 & 7.6 \\
\hline 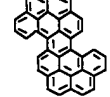 & $\begin{array}{l}\text { Dibenzo[jk,uv]dinaphtho[2,1,8,7,-defg: } 2^{\prime}, 1^{\prime}, 8^{\prime}, 7^{\prime} \text { - } \\
\text { opqr]pentacene (non planar) }\end{array}$ & $\mathrm{J}$ & 1.39 & 11 & 15.6 & 1.2 & 7.6 \\
\hline
\end{tabular}


trons or length-to-breadth ratio of the molecule. A second class of LPAHs are those which are highly non-planar because of steric hindrance produced by "fjord" substructures (Fig. 1A) [16]. Examples of this class are tetrabenzo[a,cd,j,lm]perylene $(\mathrm{H})$, and dibenzo[jk,uv]dinaphtho[2,1,8,7-dfg: $: 2^{\prime}, 1^{\prime}, 8^{\prime}, 7^{\prime}$-opqr]pentacene (J). A third class of LPAHs are those molecules whose degree of planarity is intermediate between planar and the highly non-planar structure, because they possess the "cove" substructure (Fig. 1B). Examples of this class include: dibenzo[j,lm]naphtho[1,8-ab]perylene $(\mathrm{C})$, benzo[lm]phenanthro-[4,5,6-abcd]perylene (F) and dibenzo[a,rst]naphtho[8,1,2-cde]pentaphene (I). These discoveries indicate that a stationary phase with shape selectivity will be of great value in the separation of LPAHs.

Previous research on the separation of small PAHs suggests that recently developed protoporphyrin (ProP) and metalloprotoporphyrin (MProP)-silica phases may prove useful for shape selective separation of LPAH solutes. As novel stationary phases developed for liquid chromatography, ProP-silica and MProP-silica have found applications in the separation of smaller amino acids and peptides, as well as smaller PAHs [17-19]. Different categories of solutes can be separated on ProP- and MProP-silica because of the multiple modes of interactions on such phases, including hydrophobic interaction, $\pi-\pi$ interaction, and ligation interaction [18]. In this work, the retention of LPAHs on FeProP-silica phases is evaluated and compared with other stationary phases.

\section{Experimental}

Reagents. Protoporphyrin IX (ProP) was purchased from MidCentury Chemicals (Posen, IL). Hyperprep ${ }^{\circledR} 8 \mu \mathrm{m}$ silica gel with a surface area of $90 \mathrm{~m}^{2} / \mathrm{g}$ and a pore size of $300 \AA$ was a product of Keystone Scientific (Bellefonte, PA). Aminopropyltriethoxysilane was obtained from Hüls (Piscataway, NJ). Hemin (chloroprotoporphyrin IX iron(III)), anhydrous dimethylformamide (DMF) used in the immobilization of ProP, and HPLC grade DMF, employed in the metallation of protoporphyrin-silica, were purchased from Aldrich (Milwaukee, WI). HPLC grade methanol and acetonitrile utilized as mobile phases were also purchased from Aldrich. The coupling reagent, 1,1'-carbonyldiimidazole (CDI), was a product of Fluka (Ronkonkoma, NY). Doubly deionized water was filtered through a $0.45 \mu \mathrm{m}$ polypropylene membrane from Alltech Associates (Deerfield, IL) prior to use in preparing various mobile phases. LPAH samples of dibenzo[cd,lm]perylene, dibenzo[j,lm]naphtho[1,8-ab]perylene, benzo[pqr]-naphtho[8,1,2-bcd]perylene, pyranthrene, benzo[l,m]phenanthro[4,5,6-abcd]perylene, naphtho[8,1,2-abc]coronene, tetrabenzo[a,cd,j,lm]perylene, coronene, dibenzo[a,rst]naphtho[8,1,2-cde]pentaphene, and dibenzo [jk,uv]dinaphtho[2,1,8,7-dfg:2',1', $8^{\prime}, 7^{\prime}$-opqr]pentacene were synthesized or isolated as reported previously $[1-3,10,15]$.

Preparation of the protoporphyrin and metalloprotoporphyrin silica stationary phases. A detailed procedure for synthesizing the traditional ProP-silica and MProP-silica stationary phases was described elsewhere $[17,19]$. Briefly, the silica gel was first reacted with excess of 3-aminopropyltriethoxysilane (10 times in excess of the necessary amount assuming a maximum surface coverage of $8 \mu \mathrm{mol} / \mathrm{m}^{2}$ ) in toluene to obtain the aminopropyl-silica. ProP ( 5 times in excess of the amine coverage on silica surface after the 3-aminopropyltriethoxysilane treatment) was then activated with CDI (2 equivalents per ProP) in DMF, and reacted with the amino- propyl-silica to form ProP-silica via an amide bond between the porphyrin and the surface amine groups. The excess amine groups were then end-capped by refluxing the silica gel with acetic anhydride. Each of the phases was then metallated with $\mathrm{Fe}(\mathrm{III})$ by refluxing with $\mathrm{FeCl}_{3} \cdot 6 \mathrm{H}_{2} \mathrm{O}$ (10 times in excess of the immobilized porphyrin) in $50 \mathrm{~mL}$ DMF for $4 \mathrm{~h}$. Surface coverage was determined by percentage increase in nitrogen content obtained from $\mathrm{CHN}$ analysis performed in house.

Instrumentation and chromatographic conditions. The HPLC system consisted of a Spectra Physics (San Jose, California) SP 8700 solvent delivery system, a Spectra Physics SP 4290 computing integrator, a Kratos (Ramsey, New Jersey) Spectroflow 773 variable wavelength UV-Vis detector, and a Rheodyne (Cotati, California) model 7010 sample injection valve with a $20 \mu \mathrm{L}$ loop. An LC 305 scanning fluorescent detector from Thermo Separation Products (San Jose, California) was also used as an alternative detector. Capacity factors of LPAHs were measured using $1.0 \mathrm{~mL} / \mathrm{min}$ flow rate unless otherwise stated. Fluorescence detection was employed and a Shimadzu spectrofluorometer RF-1501 was used to determine the optimal wavelengths for LPAHs prior to HPLC experiments. The excitation wavelengths ranged from $310 \mathrm{~nm}$ to $330 \mathrm{~nm}$ and the emission wavelengths were from $440 \mathrm{~nm}$ to $480 \mathrm{~nm}$.

\section{Results and discussion}

Unique shape selective retention of LPAHs on ProP-silica phases

Fetzer and Biggs [15] investigated the retention behavior of LPAHs on various bonded phases and have concluded that the nature of the stationary phase is very important with respect to the final separation. The characteristics of given LPAHs are also important to the retention observed, with the planarity of the LPAH being one of the most important factors. Three distinct retention behaviors can be linked to the three classes of molecular shape: fjord, cove, and bay (Fig. 1). Previous studies of ProP- and MProP-silica phases have demonstrated that these phases have very strong shape selectivity for the separation of smaller PAHs [17]. Thus, ProP-silica should also be suitable for LPAH separations as well.

To test the shape selectivity of ProP-silica toward the retention of LPAHs, the capacity factors of $10 \mathrm{LPAHs}$ with different size and planarity were examined on columns packed with FeProP-silica (Table 1). The capacity factors were measured on a Fe(III)ProP-silica phase with porphyrin coverage of $0.2 \mu \mathrm{mol} / \mathrm{m}^{2}$ using a flow rate of $1.0 \mathrm{~mL} / \mathrm{min}$. The shape of the compounds is generally described as planar, twisted or non-planar. In a planar compound, the steric hindrance comes from a "bay" substructure, whereas in a twisted compound, the hindrance comes from a "cove" substructure, and in a non-planar one, the hindrance comes from a "fjord" substructure or two adjacent cove substructures (Fig. 1 A, B and C). As shown in Table 1 the planar molecules exhibit significantly larger capacity factors than the non-planar LPAHs (e.g., compound $\mathrm{G}$ and $\mathrm{H}$ ). A different degree of twist in the molecule also results in different capacity factors. When using $100 \%$ acetonitrile (ACN) as mobile phase, for example, the capacity factor of compounds that have 9 rings (compounds F, G, H and I) ranged from 4.0 to 62 de- 
(A)

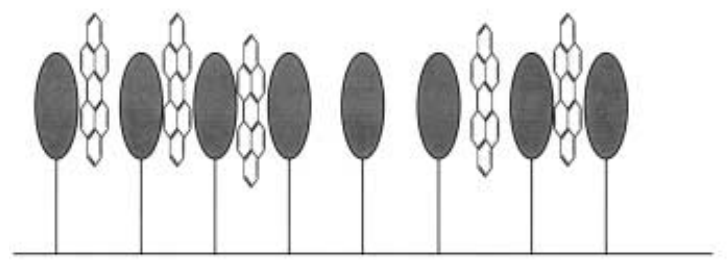

(B)

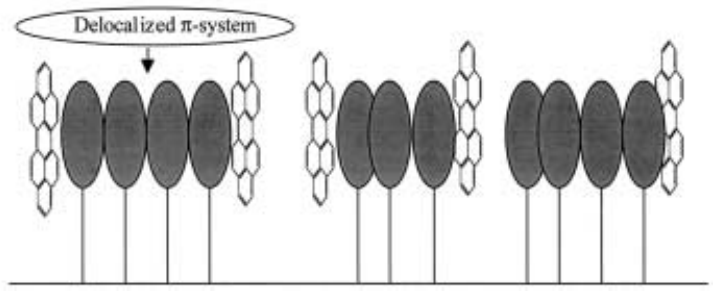

Fig. 2 Schematic diagram of slot mechanism (A) and cluster mechanism $(B)$ for porphyrin-silica stationary phases

pending on their shape. The capacity factor of compound $\mathrm{H}$ is only 4.0 because it has a highly non-planar structure, while compounds F and I almost have the same capacity factor, 25.0, because of their similarity in degree of twist. Compound $\mathrm{G}$ also has 9 rings, but exhibits a capacity factor of 62.0 because it is perfectly planar. Since the selectivity is based on the planarity of the molecule, the interaction mechanism of LPAHs on ProP-silica could potentially be analogous to the slot mechanism first proposed by Wise and Sander [20] (see Fig. 2 A). After immobilization, porphyrins may form planar $\pi$-rich slots on silica surface as described previously [17]. Due to the planarity of these slots, only planar LPAHs can slide into the space between adjacent ProP species and can achieve a favorable face-to-face interaction with the immobilized porphyrin or porphyrins. The twisted LPAHs can only slide partially into the slots, thus their interaction is weaker and the capacity factor smaller than for the planar species. In the case of non-planar LPAHs, it would be difficult for these solutes to slide into the slots, and therefore the interaction between the molecule and the immobilized porphyrin may only occur at the rim of the porphyrin macrocycle. Hence, the $\pi-\pi$ interactions are minimal and thus, these non-planar compounds exhibit the shortest retention among the three classes of compounds examined. Partial evidence for a possible slot mechanism with respect to PAH retention on ProP-silica phases was suggested previously [17], when it was shown that for smaller PAH solutes the planar/non-planar shape selectivity was significantly enhanced when a higher density of ProP species was immobilized on the surface of the silica.

Although a slot mechanism provides one possible explanation for the experimental data for LPAH retention obtained on ProP-silica phases, the packing materials used in this particular study had a relatively low surface density of porphyrins $\left(0.2 \mu \mathrm{mol} / \mathrm{m}^{2}\right)$. Thus, the possibility of forming slots, where two immobilized porphyrin species would be just the right distance apart to interact simultaneously with a given LPAH solute (Fig. 2 A), seems unlikely. It is, however, well known that porphyrins have a strong tendency to form $\pi-\pi$ aggregates. Therefore, during the immobilization reaction, it is potentially easier for porphyrin molecules to form aggregates than to form evenly spaced slots after immobilization, even when the total final surface coverage of porphyrin species is quite low. This would result in a surface layer of immobilized porphyrins that is rather heterogeneous, but one where clusters of immobilized porphyrin aggregates are spaced out over the surface of the silica (see Fig. 2B). The distance between porphyrin molecules within a given immobilized aggregate would be too small even for a planar PAH to slide in, but an LPAH molecule can interact at the planar edge of such porphyrin clusters. It is possible that the $\pi$-orbitals from a planar LPAH molecule and the $\pi$-orbitals from the stacked porphyrin can overlap, thus, forming a strong $\pi-\pi$ complex. The delocalized $\pi$-system of the porphyrin aggregate could further make the interaction between an LPAH and the immobilized porphyrin much stronger than the interaction between a planar LPAH and an isolated single immobilized porphyrin species (although the latter is also clearly possible). For the non-planar LPAHs, their interaction with the immobilized porphyrin cluster tends to be weaker due to a smaller face-toface overlap for the $\pi-\pi$ interaction. Evidence for the presence of these clusters/aggregates of porphyrins on the surface influencing the shape selectivity again comes from earlier studies in which it was clearly shown that planar solute selectivity is enhanced when stationary phases with a higher surface coverage of the ProP species are used [17], and more recent spectroscopic studies of the ProPsilica materials [21]. In the latter experiments, it was found that porphyrins immobilized by the method described herein have much broader UV-Vis absorption bands (Q-bands) compared to ProP-silica phases in which the ProP species were intentionally spread out and prevented from being immobilized as aggregates on the silica surface [21]

In the present work, it was found that the retention time for planar LPAHs on FeProP-silica phases were so long (> $60 \mathrm{~min}$ ) that it is impossible to finish the experiments within reasonable time using hexane as the mobile phase (data not shown). For the same planar LPAHs, the capacity factors decrease dramatically $(<6.4)$ in $100 \%$ toluene (a $\pi$-rich solvent), indicating that $\pi$ - $\pi$ interaction is, in fact, the predominant retention mechanism. Indeed, the mixture of toluene/acetonitrile (or toluene/hexane) can be used to tune the capacity factors to a suitable range in order to achieve a sufficient separation (see Table 1).

Introducing the length to breadth ratio $(\mathrm{L} / \mathrm{B})$ can help explain the shape selective retention of LPAHs on FeProP-silica more clearly. The $\mathrm{L} / \mathrm{B}$ ratio is a specific shape parameter of a given LPAH molecule. The correlation of solute L/B with the retention of PAH isomers on an ordered stationary phase was first demonstrated by Janini et al. in gas chromatography (GC) using a liquid crys- 
talline stationary phase [22]. Wise et al. [23] observed the same correlation for PAH isomers on an ordered stationary phase in high-performance liquid chromatography using a polymeric ODS reversed-phase column. Since the FeProP-silica stationary phase is highly shape-selective, with a high degree of order, the L/B ratio should be a very important parameter that influences LPAH solute retention on columns packed with FeProP-silica.

There are several proposed ways to calculate L/B [23-25]. The L/B ratio used in this study was calculated by a method proposed by Wise et al. [23]. The L/B was computed graphically from the rectangle enclosing the molecule that gives the maximum $\mathrm{L} / \mathrm{B}$ ratio. There is only one way of drawing the rectangle which gives the maximum L/B ratio. The calculation was based on the assumption that the L/B ratio of a twisted molecule will not be significantly different than of a planar one; hence, only the planar representation was used. The distance between atoms and the van der Waals radii of the outer atoms of the molecule were used for these calculations as illustrated in Fig. 3, and the results of these calculations are listed in Table 1. By correlating L/B ratios of LPAHs and the shape selectivity described previously, several observations can be made from the retention data of LPAHs on FeProP-silica phases:

First, if compounds have the same number of rings, planar species are always retained longer than twisted and non-planar ones. For example, compound D, a planar compound with 8 rings has a capacity factor of 44.0,

(A)

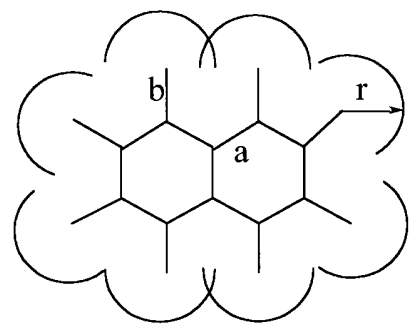

(B)
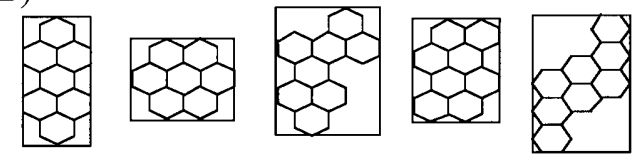

$\mathrm{L} / \mathrm{B}=1.78$

1.17

1.42

1.36
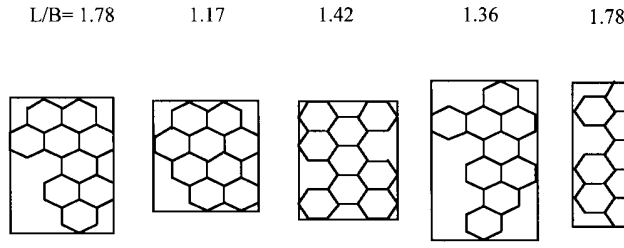

1.57

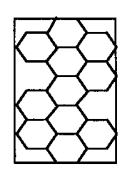

1.39

Fig. 3 Schematic of L/B calculation for LPAH solutes: (A) Different bond types used in the calculation, $a=1.4 \AA$ (C-C aromatic); $b=1.1 \AA$ (C-H single bond); $r=1.2 \AA ;(B)$ schematic diagram of the largest possible L/B values for individual LPAHs $(\mathrm{C}-\mathrm{H}$ bond and $r$ are omitted for simplicity) while compound $\mathrm{C}$, a twisted compound with 8 rings has a capacity factor of 28.0 when using $100 \%$ acetonitrile as mobile phase.

Second, if solutes have same structure but different ring number, the one with larger number of rings (i.e., higher carbon number) is retained longer, e.g., compounds A and D.

Third, if compounds have the same number of rings and are both planar, the one with the larger $\mathrm{L} / \mathrm{B}$ ratio is retained longer. For example, compound $\mathrm{A}$ and $\mathrm{B}$ both are planar LPAHs with 7 rings and using $100 \%$ acetonitrile as the mobile phase, $\mathrm{k}_{\mathrm{A}}=13.4$ and $\mathrm{k}_{\mathrm{B}}=11.0$. The same trend was observed for compounds D and E, two planar LPAH with 8 rings; compound $\mathrm{D}$ has a smaller capacity factor $\left(\mathrm{k}_{\mathrm{D}}=44.0\right)$ and smaller L/B (1.36) compared with compound $\mathrm{E}\left(\mathrm{k}_{\mathrm{E}} 49.0\right.$ and $\left.\mathrm{L} / \mathrm{B}=1.78\right)$. This can be explained by the fact that the $\pi-\pi$ interaction of porphyrin is peripheral, so that LPAHs will tend to overlap with the porphyrin ring at its "length" side rather than the "breadth" side for maximum interaction. LPAHs with larger L/B will have slightly longer "length", and therefore stronger interaction and larger capacity factors than LPAHs with smaller L/B.

Fourth, if compounds have same number of rings and are twisted by having a "cove" substructure, the capacity factor will be very similar since the "footprint" of these compounds on the immobilized porphyrin will be nearly the same despite of their differences in $\mathrm{L} / \mathrm{B}$ value. This is because there is no direct face-to-face $\pi$ - $\pi$ interaction if the molecule is twisted. Only part of the molecule can interact with the immobilized porphyrin. An example of such a case is compounds F and I, two 9-ring LPAHs, with both of them having one cove substructure and being twisted. The capacity factors of the two compounds are very similar under all mobile phase conditions: when $100 \%$ acetonitrile was used as mobile phase, $\mathrm{k}_{\mathrm{F}}=25$ and $\mathrm{k}_{\mathrm{I}}=24.0$; when the mobile phase was $80 \% / 20 \%$ acetonitrile/toluene, $\mathrm{k}_{\mathrm{F}}=7.6$ and $\mathrm{k}_{\mathrm{I}}=7.2$.

Fifth, if an LPAH(1) has $\mathrm{n}$ rings and is planar, and LPAH(2) has $n+1$ rings but is non-planar, it is difficult to determine the elution sequence from the planarity rule or the carbon number rule because the one that is planar also has the lower number of carbon atoms. In this case, the elution order has to be determined by experiments. For example, compound $\mathrm{F}$ is a twisted LPAH with 9 rings and compound $\mathrm{D}$ is a planar LPAH with 8 rings, the capacity factors are 25.0 and 28.0, respectively, with compound D (planar) exhibiting a slightly longer retention. The sequence is different in the case of compound $\mathrm{A}$ and $\mathrm{C}$ with compound $\mathrm{C}$ being a twisted LPAH with 8 rings and compound $\mathrm{A}$, a planar molecule with 7 rings. Indeed, compound $\mathrm{C}$ (twisted) has a larger capacity factor $(28.0 \mathrm{vs}$. 13.4 in $100 \%$ acetonitrile).

It is also shown in Table 1 that the retention sequence of LPAHs on FeProP-silica will not change by changing the solvent, i.e., if $\operatorname{LPAH}(1)$ is retained longer than LPAH(2) in acetonitrile, it will also be retained longer than LPAH(2) when using toluene or a mixture of acetonitrile and toluene or any other solvent (such as hexane 
or dichloromethane (data not shown)) on the FeProP-silica phase. Thus, the retention sequence of LPAHs on FeProP-silica appears to be more predictable than on other reversed-phase columns [16]. Such retention order information is very useful in the correct assignment of chromatographic peaks, which tends to be a challenging task when analyzing LPAH isomers.

Comparison of LPAH retention on FeProP-silica with other stationary phases

Reversed-phase liquid chromatography (RPLC) has been used successfully to separate LPAHs previously. Researchers have studied the retention of LPAHs on various bonded stationary phases, including monomeric ODS, polymeric ODS, diphenyl-silica and pyrene-silica phases, which are popular reversed-phase materials for HPLC. Their studies on the elution behavior of LPAHs indicate that LPAH retention is controlled by two main factors: (1) orderliness of the bonded phase and (2) degree of solute planarity [26]. Comparing the retention of LPAHs on ProP-silica and other stationary phases can generate useful information about the differences between these phases and also aid in understanding the retention mechanism of LPAHs on ProP-silica phases.

The capacity factors of several LPAHs on different stationary phases are listed in Table 2. ODS1 is a polymeric phase, ODS2 is monomeric ODS phase, and ODS3 is monomeric ODS end-capped with methyl groups. NE stands for naphthylethyl-silica and PE stands for pyrenylethyl-silica [25]. The FeProP-silica evaluated for this comparison study was synthesized in-house using a "spread" method with low surface coverage $\left(0.22 \mu \mathrm{mol} / \mathrm{m}^{2}\right)$ [21]. Normally, the surface coverage of FeProP on silica can range from 0.2 to $3.0 \mu \mathrm{mol} / \mathrm{m}^{2}$. A lower surface coverage of FeProP-silica phase was used in this study because the capacity factors for the planar LPAHs would be too long if a high surface coverage phase was employed. Given the fact that the capacity factors of LPAHs on FeProP-silica were obtained with a much stronger mobile phase $(80 / 20$ dichloromethane/methanol) than on other stationary phases (15/85 dichloromethane/methanol), the data presented in Table 2 actually indicate that the capacity factors of LPAHs on FeProP-silica phase would be much larger than on other common reversed-phase columns if the same mobile phase was used. It has been observed by other researchers that when using 75/25 dichloromethane/methanol as the mobile phase, the capacity factors of the LPAHs listed in Table 2 are less than 1.00 on most of the stationary phases except pyrenylethyl(PE)-silica [26]. In contrast, on the FeProP-silica phase using a slightly stronger mobile phase (80/20 dichloromethane/methanol), the capacity factors of the LPAHs tested are in the range of 1-25. This indicates that the capacity factors of LPAHs on ProP-silica have a much greater range than other reversed-phase materials. Thus, a two-solvent-gradient should be sufficient for the separation of these solutes, while on other conventional reversed-phase materials, usually a three solvent system must be employed for separation of LPAHs [15].

On the polymeric ODS1 phase, the capacity factors of LPAHs increase with the ring number (see Table 3). For example, compounds with 7 rings (A and B) have smaller capacity factors than those with 8 rings (D), and LPAHs with 8 rings (D) exhibit smaller capacity factors than ones with 9 rings $(\mathrm{G}$ and $\mathrm{H})$. There is some degree of shape selectivity since compound $\mathrm{G}$ (planar) has a larger capacity factor than $\mathrm{H}$ (non-planar). On monomeric ODS2, similar trends are observed except that the planarity selectivity appears to be higher since the capacity factor of compound $\mathrm{D}$ (planar with 8 rings) is larger than that of compound $\mathrm{H}$ (non-planar with 9 rings). On monomeric ODS3, the capacity factors of LPAHs increase with increasing ring number, very similar to the ODS1 phase. However, it was noted by Fetzer and coworkers [16] that the retention sequence of LPAHs on ODS changes in different mobile phase solvents. For this reason, the LPAH retention behavior on ODS phases listed in Table 3 cannot be used as an indication of their retention behavior in other mobile phases. This is in distinct contrast to the retention behavior found on FeProP-silica phases, where the LPAH retention does not change with a change of mobile phase. On the naphthylethyl phase, for example, it appears that the LPAHs are eluted in the order of increasing number of rings as well, i.e., compounds $\mathrm{A}$ and $\mathrm{B}$ have smaller capacity factors than $\mathrm{D}$, and $\mathrm{D}$ has a smaller capacity factor than $\mathrm{H}$. There is no shape selectivity observed on the naphthylethyl phase, since $\mathrm{k}_{\mathrm{H}}=4.09$ while $\mathrm{k}_{\mathrm{G}}=3.64$. On the pyrenylethyl (PE) phase, the capacity factors of the

Table 2 Capacity factor of LPAHs on different stationary phases

\begin{tabular}{|c|c|c|c|c|c|c|c|c|c|c|}
\hline & Rings & Symbol & $\mathrm{L} / \mathrm{B}$ & Shape & ODS $1^{\mathrm{a}}$ & ODS2 $2^{\mathrm{a}}$ & $\begin{array}{l}\mathrm{ODS}^{\mathrm{a}} \\
\text { Capaci }\end{array}$ & $\begin{array}{l}\mathrm{NE}^{\mathrm{a}} \\
\text { ty factors }\end{array}$ & $\mathrm{PE}^{\mathrm{a}}$ & FeProPb \\
\hline Coronene & 7 & $\mathrm{~B}$ & 1.17 & planar & 2.43 & 5.33 & 3.23 & 1.34 & 6.47 & 3.45 \\
\hline Dibenzo[cd,lm]perylene & 7 & A & 1.78 & planar & 3.02 & 7.9 & 4.39 & 1.75 & 10.9 & 4.71 \\
\hline Benzo[ghi]naphtho-[8,1,2-cde] perylene & 8 & $\mathrm{D}$ & 1.36 & planar & 4.87 & 14.6 & 6.38 & 2.48 & 28.1 & 16.7 \\
\hline Tetrabenzo[a,cd,j,lm]-perylene & 9 & $\mathrm{H}$ & 1.28 & non-planar & 5.78 & 10.7 & 7.55 & 4.09 & 21.6 & 1.28 \\
\hline Naphtho[8,1,2-abc]-coronene & 9 & G & 1.18 & planar & 8.41 & $>14.6$ & 10.6 & 3.64 & $>28.1$ & 20.3 \\
\hline
\end{tabular}

ODS 1: polymeric; ODS2: monomeric; ODS3: monomeric with $\mathrm{C}_{1}$; NE: naphthyl-ethyl; PE: pyrenyl-ethyl; FeProP: iron protoporphyrin.

a mobile phase: 15/85 dichloromethane/methanol [25];

${ }^{\mathrm{b}}$ mobile phase: $80 / 20$ dichloromethane/methanol 
LPAHs follow the same trend as on ODS-silica, but their absolute values are significantly larger because of the stronger $\pi-\pi$ interactions between the LPAH solutes and the immobilized pyrene.

On FeProP-silica phases, the shape selectivity overwhelms the size of the molecule unlike any other reversed-phase material. This is likely due to the fact that the bonded porphyrin is highly ordered because of the face-to-face $\pi-\pi$ interaction between adjacent immobilized porphyrins [17, 19] (see Fig. 2 B, above). For example, compound $\mathrm{H}$ is a non-planar LPAH with 9 rings, while compound A is a planar LPAH with 7 rings. The retention of $\mathrm{H}$ is larger than that of compound $\mathrm{A}$ on a pyrene phase, indicating that the carbon number is the dominant factor in retention, not the planarity. On the FeProP-silica phase, the retention of compound $\mathrm{A}$ is significantly longer than that of compound $\mathrm{H}$ although $\mathrm{A}$ is smaller in size, indicating that planarity is the predominant factor. Similar examples include compounds $\mathrm{F}$ and $\mathrm{D}$.

A comparison of the shape selectivity of the different stationary phases is listed in Table 3 . The selectivity factor $(\alpha)$ of dibenzo[cd, lm]perylene/coronene (A/B) is an indication of topology selectivity while the selectivity factor of naphtho[8,1,2-abc]coronene/tetrabenzo[a,cd,j,lm]perylene is an indication of planarity selectivity. The ODS phases all have similar topology selectivity, with $\alpha_{\mathrm{A} / \mathrm{B}}$, being $1.24,1.48,1.36$ for the three phases. Further, the planarity selectivity of these phases is also similar with $\alpha_{\mathrm{G} / \mathrm{H}}$ being 1.46, 1.36, 1.40 for the three ODS phases. The NE phase also has similar topology selectivity as the ODS phases, but does not demonstrate similar planarity selectivity. In fact, the selectivity for planar LPAHs is smaller than 1.0, indicating a non-planarity selectivity behavior for NE phases. The PE phase has better topology selectivity than the ODS phases but similar planarity selectivity. FeProP-silica demonstrates the highest planarity selectivity among all phases, with $\alpha_{\mathrm{G} / \mathrm{H}}$ being 15.9, much higher than any other reversed-phase columns.

Table 3 Comparison of shape selectivity of LPAHs on different stationary phases

\begin{tabular}{lllllll}
\hline & ODS1 $^{\mathrm{a}}$ & ODS2 $^{\mathrm{a}}$ & ODS3 $^{\mathrm{a}}$ & $\mathrm{NE}^{\mathrm{a}}$ & PE $^{\mathrm{a}}$ & FeProP $^{\mathrm{b}}$ \\
\hline$\alpha_{\mathrm{A} / \mathrm{B}}$ & 1.24 & 1.48 & 1.36 & 1.30 & 1.68 & 1.37 \\
$\alpha_{\mathrm{G} / \mathrm{H}}$ & 1.46 & $>1.36$ & 1.40 & 0.89 & $>1.28$ & 15.9 \\
\hline
\end{tabular}
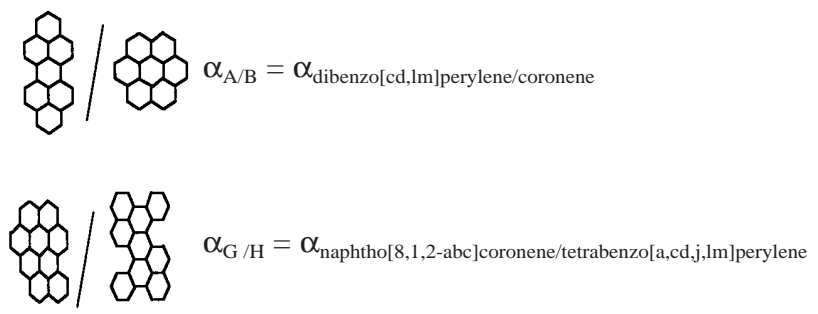

ODS1: polymeric; ODS2: monomeric; ODS3: monomeric with $\mathrm{C}_{1}$;NE: naphthylethyl; PE: pyrenyl-ethyl; FeProP: iron protoporphyrin.

a mobile phase: 15/85 dichloromethane/methanol [25];

${ }^{\mathrm{b}}$ mobile phase: 80/20 dichloromethane/methanol
This indicates that on FeProP-silica phase, the shape of the molecule is a far more important factor in retention than the molecular weight. With $\alpha_{\mathrm{A} / \mathrm{B}}$ being 1.37 , the topology selectivity on FeProP-silica seems somewhat smaller than on all other reversed-phase materials. Indeed, considering the fact that the FeProP-silica has a surface coverage of only $0.22 \mu \mathrm{mol} / \mathrm{m}^{2}$, and since the selectivity highly depends on the surface coverage, using a higher surface coverage FeProP-silica material may enhance the topology selectivity for LPAH solutes, as was observed in the case of the smaller PAHs examined in earlier work [17].

\section{Conclusions}

FeProP-silica phases exhibit unique shape selectivity for LPAH retention, with the planar LPAHs always being retained much longer than the non-planar ones. Solute planarity, L/B value, and carbon number all contribute to the retention of LPAHs on ProP-silica. It has been found that the retention of LPAH solutes on columns packed with FeProP-silica is more predictable than other reversedphase columns. Thus, by varying the toluene or dichloromethane content in the mobile phase, the capacity factors of LPAHs can be tuned to a range required for a given analysis, and at the same time, the retention order of LPAHs will remain the same. Due to the very high planarity selectivity of FeProP-silicas, it should be possible to use these phases in conjunction with columns packed with other materials to devise more efficient methods for the HPLC separation of complex mixtures of LPAHs in environmental and other samples.

\section{References}

1. Fetzer JC, Biggs WR, Jinno K (1986) Chromatographia 21:439

2. Sullivan RF, Boduszynski MM, Fetzer JC (1989) Energy Fuels 3:603

3. Fetzer JC, Rechsteiner CE (1991) Polynuclear Aromatic Hydrocarbons: Eleventh International Symposium. Eds: Cooke M, Loening K, Merritt J, Battelle Press, Columbus, OH.

4. Fetzer JC, Kershaw JR (1995) Fuel 74:1533

5. Harvey RG (1991) Polycyclic Aromatic Hydrocarbons: Chemistry and Carcinogenicity, Cambridge University Press, New York, Ch3.

6. Aoki J, Takekawa M, Fujisawa S, Iwashima S (1977) Bull Chem Soc Japan 50:1017

7. Brindle R, Albert K (1997) J Chromatogr 753:3

8. Sander LC, Wise SA (1995) Anal Chem 67:3284

9. Kimata K, Hirose T, Moriuchi K, Kosoya K, Araki T, Tanaka N (1995) Anal Chem 67:2556

10. Grosse-Rhode C, Kicinski HG, Kettrup (1990) Chromatographia 29:489

11. Yanaka N, Tokuda Y, Iwaguchi K, Araki M (1982) J Chromatogr 239:761

12. Verzele M, Van de Velde M (1985) Chromatographia 20:239

13. Sander LC, Parris R, Wise SA (1991) Anal Chem 63:2589

14. Smith TR, Strickler VA (1980) J High Resolut Chromatogr, Chromatogr Commun 3:634

15. Fetzer JC, Biggs WR (1985) J Chromatogr 346: 81 
16. Fetzer JC (1988) Polynuclear Aromatic Compounds, Advances in Chemistry. Ed: Ebert LB, vol 217, American Chemical Society, Washington, DC, Ch 17.

17. Chen S, Meyerhoff M (1998) Anal Chem 70:2523

18. Kibbey CE, Meyerhoff ME (1993) Anal Chem 65:2189

19. Xiao J, Kibbey CE, Coutant DE, Martin GB, Meyerhoff ME (1996) J Liquid Chrom Rel Technol 19(17\&18):2766

20. Wise SA, Sander LC (1985) J High Resolut Chromatogr, Chromatogr Commun 8:248

21. Chen S, Ruedel U, Meyerhoff M (1999) J Chromatogr 859:121
22. Janini GM, Johnston K, Zielinski WL (1975) Anal Chem 47:670

23. Wise SA, Bonnett WJ, Guenther FR, May WE (1981) J Chromatogr Sci 19:457

24. Radecki A, Lamparczyk H, Kaliszan R (1979) Chromatographia 12:595

25. Lamparczk H, Radecki A, Kaliszan R (1981) Biochem Pharmacol 130:2337

26. Jinno K, Nagoshi N, Tanaka N, Okamoto M, Fetzer JC, Biggs WR (1987) J Chromatogr 386:123 